\title{
Pulseira fotográfica como ferramenta inovadora no protocolo de identificação do paciente com transtorno mental agudo
}

\author{
Photographic bracelet as an innovative tool in the identification protocol for patients with acute mental \\ disorder
}

\author{
Pulsera fotográfica como herramienta innovadora en el protocolo de identificación del paciente con \\ transtorno mental agudo
}

\author{
Andreia Novais Dias Blanco'; Renata Flavia Abreu da Silva", \\ Vanessa de Almeida Ferreira Corrêa ${ }^{\prime \prime \prime}$; Amanda Rebeca de Oliveira Rebelo ${ }^{\text {IV }}$
}

\begin{abstract}
RESUMO
Objetivo: descrever a elaboração de protocolo para a identificação do paciente com transtorno mental agudo. Método: estudo exploratório e qualitativo, realizado de maio 2018 a janeiro 2019, por meio das etapas: revisão integrativa da literatura, questionário online respondido por 17 profissionais de saúde vinculados à Sociedade Brasileira para a Qualidade do Cuidado e Segurança do Paciente, e grupo focal com 04 especialistas em Saúde Mental. Para tratamento dos dados, utilizou-se a análise descritiva e comparativa. Resultados: na revisão não se encontraram artigos sobre identificação do paciente com transtorno mental agudo. Na consulta aos especialistas da segurança do paciente identificou-se que $82,3 \%$ não possuíam em suas instituições protocolo específico. No grupo focal evidenciou-se dificuldade na identificação deste paciente. Conclusão: acreditase que o protocolo com a inserção da pulseira fotográfica apresenta-se como uma ferramenta inovadora na redução de riscos associados à identificação deste paciente.
\end{abstract}

Descritores: Transtornos Mentais; Pessoas Mentalmente Doentes; Segurança do Paciente; Sistemas de Identificação de Pacientes.

\begin{abstract}
Objective: to describe the development of a protocol for identification of patients with acute mental disorders. Method: this qualitative exploratory study was carried out from May 2018 to January 2019 through an integrative literature review, an online questionnaire answered by 17 health personnel belonging to the Brazilian Society for Quality of Care and Patient Safety, and a focus group of four mental health experts. Results: no articles specifically on identification for patients with acute mental disorders were found in the review. The consultation of patient safety experts found that $82.3 \%$ had no specific protocol in their institutions. The focal group highlighted difficulties communicating with these patients. Conclusion: the protocol including the photographic bracelet is believed to constitute an innovative tool for reducing risks associated with identification of these patients.

Descriptors: Mental Disorders; Mentally III People; Patient Safety; Patient Identification Systems.

\section{RESUMEN}

Objetivo: describir el desarrollo de un protocolo para la identificación de pacientes con trastornos mentales agudos. Método: este estudio exploratorio cualitativo se realizó de mayo de 2018 a enero de 2019 a través de una revisión integradora de la literatura, un cuestionario en línea respondido por 17 miembros del personal de salud pertenecientes a la Sociedad Brasileña de Calidad de Atención y Seguridad del Paciente, y un grupo focal de cuatro personas de salud mental expertos. Resultados: en la revisión no se encontraron artículos específicos sobre identificación de pacientes con trastornos mentales agudos. La consulta de expertos en seguridad del paciente encontró que el $82,3 \%$ no tenía un protocolo específico en sus instituciones. El grupo focal destacó las dificultades para comunicarse con estos pacientes. Conclusión: se cree que el protocolo que incluye la pulsera fotográfica constituye una herramienta innovadora para reducir los riesgos asociados a la identificación de estos pacientes. Descriptores: Transtornos Mentales; Enfermos Mentales; Seguridad del Paciente; Sistemas de Identificación de Pacientes.
\end{abstract}

\section{INTRODUÇÃO}

Estudos sobre a atenção à pacientes com transtorno mental agudo apontam para os desafios encontrados nos serviços de saúde reportados à possibilidade de incidentes devido às especificidades deste paciente ${ }^{1,2}$. Na fase aguda, o paciente com transtorno mental poderá demonstrar sua angústia e seu sofrimento, por meio de sua agressividade e alteração do seu nível de consciência ${ }^{3-5}$. Estas particularidades podem desencadear um comportamento de risco, potencializando a ocorrência de incidentes.

'Enfermeira, Mestre em Saúde e Tecnologia no Espaço Hospitalar, Hospital Universitário Gaffrée e Guinle, Rio de Janeiro, Brasil. E-mail: diasandreia1978@hotmail.com. ORCID: https://orcid.org/0000-0003-1779-4954

"Enfermeira, Doutora em Ciências, Docente da Universidade Federal do Estado do Rio de Janeiro, Brasil. E-mail: rflavia@gmail.com. ORCID: https://orcid.org/0000-0003-1776-021X

I'Enfermeira, Doutora em Enfermagem, Docente da Universidade Federal do Estado do Rio de Janeiro, Brasil. E-mail: vanessa.correa@unirio.br. ORCID: https://orcid.org/0000-0001-7121-4493

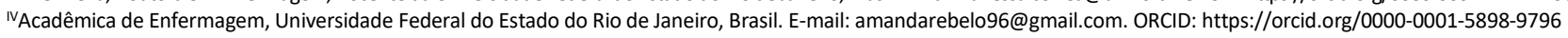


Os desafios relacionam-se às práticas dos profissionais de saúde voltadas à segurança do cuidado devido às especificidades próprias do paciente com transtorno mental agudo. Assim como, a necessidade de garantir a qualidade destas práticas em todos os serviços de saúde, no que tange a uma maior vigilância diante da imprevisibilidade deste paciente ${ }^{1}$ e à ausência de protocolo específico para a identificação desta clientela.

No sentido de promover intervenções que atendam às especificidades do paciente com transtorno mental agudo com segurança, destaca-se a sua identificação como uma importante prática inicial. A Identificação Correta do Paciente é considerada uma ferramenta essencial na prevenção e redução do dano ao paciente, que serve para garantir a qualidade do cuidado 6 .

A necessidade de Identificação Correta do Paciente está presente no Protocolo de Identificação do Paciente do Ministério da Saúde (MS), o qual apresenta ações para operacionalizar este processo ${ }^{7}$. Todavia, destaca-se que estas ações são generalistas e, por não abordarem questões próprias desta clientela, tendem a serem inefetivas.

Para a Identificação Correta do Paciente é imprescindível perguntá-lo o seu nome e confirmar por meio de pulseira instalada na sua admissão. Contudo, em pacientes com transtorno mental agudo, na ocorrência de atitudes desorganizadas, devido à alteração do pensamento e da percepção ${ }^{8}$, está intrínseca a impossibilidade da auto identificação. Isso pode colaborar para a identificação inadequada, aumentando os riscos de incidentes nos momentos necessários de sua identificação, tendo como exemplo de risco de incidente, a administração de medicamentos.

Destaca-se que identificar corretamente o paciente é a garantia que o tratamento será realizado na pessoa certa, previne os riscos de incidentes e diminui as chances de erros, os quais podem ocorrer em qualquer momento da internação. No contexto da psiquiatria, observa-se dificuldades na aplicação prática do que é proposto pelas diretrizes do protocolo de segurança do paciente ${ }^{6}$, sendo necessário construir conhecimento científico, além de propostas inovadoras voltadas à temática.

Assim, o objetivo deste estudo é descrever a elaboração de protocolo para a identificação do paciente com transtorno mental agudo.

\section{REFERENCIAL TEÓRICO}

Segurança do Paciente é uma temática que tem permeado discussões no mundo há pouco mais de duas décadas e, de forma mais sistematizada no Brasil a partir de 2013, devido à publicação do Programa Nacional de Segurança do Paciente (PNSP). Desde a sua publicação, o PNSP tem privilegiado os esforços em prol do alcance das metas nacionais, sendo a primeira: Identificar os Pacientes Corretamente ${ }^{9}$.

No intuito de atender a esta demanda, muitas iniciativas foram aperfeiçoadas e outras iniciadas, destacando-se, neste contexto, os protocolos. A partir destes, as instituições buscam qualidade em seus serviços, garantindo a segurança dos profissionais de saúde e, principalmente dos pacientes. Ao gerenciar e organizar o trabalho, podem ser considerados um elemento norteador, ou seja, um instrumento educativo ${ }^{10}$.

Os protocolos assistenciais são instrumentos que visam uniformizar a conduta dos profissionais envolvidos na assistência à saúde. Agregam aumento na segurança para o paciente e para o profissional; e auxiliam na tomada de decisão e na incorporação de novas tecnologias ${ }^{11}$. Possuem, também, como objetivo, favorecer a elaboração de indicadores de processos e resultados, aperfeiçoando a qualidade do atendimento ${ }^{12}$.

Para elaborar um protocolo, necessita-se, além de recursos e trabalho em equipe, o conhecimento do perfil epidemiológico da clientela e suas prioridades. Logo, o protocolo, deverá ser adequado à realidade do local no qual será inserido ${ }^{13}$.

Neste estudo, a inovação proposta tem relação direta com a melhoria de métodos, processos, produtos e organização do trabalho. Tal melhoria deverá levar em consideração a instituição de saúde e os pacientes nela atendidos, de modo que haja vantagem para ambos ${ }^{14}$.

Entende-se que, os riscos associados à assistência em saúde potenciais devem ser detectados e minimizados precocemente utilizando-se, para tanto, estratégias inovadoras que, quando inseridas em uma situação específica, permitem a elaboração de produtos que tendem a melhorar a prática assistencial ${ }^{15}$.

Considerando a problemática em questão, acredita-se que um protocolo de identificação do paciente, no contexto da psiquiatria, possa colaborar com a qualidade deste cuidado e, consequentemente, com a sua segurança por meio da mitigação dos riscos associados a este processo.

\section{MÉTOdo}

Estudo exploratório e de abordagem qualitativa, realizado entre maio de 2018 a janeiro de 2019 e dividido em três fases, a saber: Revisão Integrativa de Literatura; Questionário on-line com a participação de 17 profissionais de 
saúde vinculados à Sociedade Brasileira para a Qualidade do Cuidado e Segurança do Paciente (SOBRASP); e Grupo focal com quatro especialistas em Saúde Mental. Todos os participantes da pesquisa expressaram concordância em participar do estudo por meio da anuência ao Termo de Consentimento Livre e Esclarecido. O projeto de pesquisa foi aprovado pelo Comitêde Ética em Pesquisa, número do CAAE: 96664318.0.0000.5285 e número do parecer 2.854.164 de 2018.

A fase 1 desenvolveu-se com base em Revisão Integrativa de Literatura e fundamentou a discussão do tema sobre a Identificação Correta do Paciente com transtorno mental agudo no contexto da Segurança do Paciente e psiquiatria. Teve objetivo de verificar as evidências científicas do processo de identificação e assistência em saúde destes pacientes.

Na fase 2, participaram especialistas na área de Segurança do Paciente, por meio de um questionário, elaborado no Google Forms ${ }^{\circledR}$, enviado on-line via aplicativo multiplataforma de mensagens instantâneas, sendo estruturado a partir das seguintes temáticas: existência de protocolo acerca do correto processo de identificação de pacientes com transtorno mental na fase aguda nos locais de trabalho; agentes facilitadores e dificultadores na identificação destes pacientes; e necessidade de identificação dos referidos pacientes.

Os critérios de inclusão dos participantes da fase 2 foram: profissionais da área de segurança, membros da SOBRASP, atuantes em Núcleos de Segurança do Paciente, Gerência de Risco ou Qualidade. A SOBRASP foi selecionada por disseminar práticas de segurança no cuidado e reunindo profissionais interessados na temática em diferentes estados brasileiros.

Finalmente, na fase 3, realizou-se a técnica de coleta de dados, denominada grupo focal, com enfermeiros especialistas em Saúde Mental. Assim, discutiram-se as temáticas, a seguir: práticas cotidianas para identificação do paciente com transtorno mental na fase aguda; práticas alternativas de identificação deste paciente; e vivência na identificação do paciente durante a prática assistencial. Todas as fases subsidiaram a proposta do produto acadêmico confeccionado, a pulseira de identificação e têm sua síntese demonstrada na Figura 1.

\begin{tabular}{|c|c|c|}
\hline $\begin{array}{c}\text { FASE } 1 \\
\text { Revisão Integrativa }\end{array}$ & $\begin{array}{c}\text { FASE } 2 \\
\text { Profissionais de Saúde em } \\
\text { Segurança do Paciente }\end{array}$ & $\begin{array}{c}\text { FASE } 3 \\
\text { Grupo Focal (Profissionais } \\
\text { de Saúde em Psiquiatria) }\end{array}$ \\
\hline $\begin{array}{l}\text { Busca nos portais Biblioteca Virtual em Saúde (BVS) e } \\
\text { Public/Publisher Medline (PubMed), utilizando os des- } \\
\text { critores: Sistema de identificação de pacientes; segu- } \\
\text { rança do paciente; transtornos mentais; paciente psi- } \\
\text { quiátrico; pessoas mentalmente doentes; transtornos } \\
\text { psicóticos e emergência psiquiátrica, de artigos cien- } \\
\text { tíficos publicados de } 2013 \text { a setembro de } 2018 \text {. Após } \\
\text { análise dos estudos, a amostra foi de seis artigos que } \\
\text { foram classificados conforme o seu nível de evidência. }\end{array}$ & $\begin{array}{l}\text { Questionário elaborado no Google } \\
\text { Forms e enviado via WhatsApp }{ }^{\circledR} \text { a } \\
\text { membros do grupo SOBRASP, com } \\
\text { perguntas abertas abordando a } \\
\text { experiência no manejo de paci- } \\
\text { entes com transtorno mental agu- } \\
\text { do, barreiras e facilidades no pro- } \\
\text { cesso. }\end{array}$ & $\begin{array}{l}\text { Moderado pela pesquisa- } \\
\text { dora principal e norteado } \\
\text { por perguntas reflexivas so- } \\
\text { bre o processo de identifi- } \\
\text { cação de pacientes com } \\
\text { transtorno mental agudo. }\end{array}$ \\
\hline
\end{tabular}

FIGURA 1: Fases metodológicas percorridas no desenvolvimento do estudo. Rio de Janeiro, RJ, Brasil, 2019.

Os critérios de inclusão dos participantes foram: profissionais especialistas na área de Saúde Mental que possuíssem experiência prática nesta área. A coleta de dados ocorreu na Escola de Enfermagem Alfredo Pinto, na Universidade Federal do Estado do Rio de Janeiro, com duração de uma hora. Os áudios do grupo focal foram gravados e transcritos textualmente para facilitar a análise dos dados. Identificou-se os participantes do grupo focal pela categoria profissional seguida por letras maiúsculas, em ordem alfabética.

\section{RESULTADOS E DISCUSSÃo}

Na fase 1, referente à Revisão Integrativa de Literatura, selecionou-se seis artigos, os quais referenciavam a segurança do paciente, qualidade no cuidado e transtorno mental agudo. Dentre os artigos selecionados, dois destes destacaram a avaliação dos riscos aos quais os portadores de transtornos mentais estão expostos ${ }^{3,5}$. Identificou-se, também, a evidência de dano e mortalidade em internações clínicas ou cirúrgicas em pessoas com doença mental grave $^{16}$, além da percepção pelos próprios usuários de saúde mental quanto aos cuidados seguros ${ }^{17}$. A Segurança do Paciente foi vista em um artigo com foco no paciente em internação psiquiátrica ${ }^{2}$ e outro a mostrou nas publicações científicas que remetiam aos serviços comunitários de saúde mental ${ }^{4}$. 
Salienta-se que nenhum dos artigos identificados, embora atendessem à pergunta de pesquisa, apresentaram a especificidade da Identificação Correta do Paciente com transtorno mental na fase aguda no contexto da Segurança do Paciente, dificultando a identificação de evidências científicas sobre a temática, protocolos implementados e experiências nesta temática. Assim, destaca-se a importância de elaboração de ferramentas inovadoras, a partir do que se identifica como um problema no cotidiano de atenção à saúde e como lacunas do conhecimento científico para a melhoria dos processos e da qualidade do cuidado em saúde ${ }^{14}$.

No que se refere à segunda fase deste estudo, participaram 13 enfermeiros, dois médicos, um técnico de enfermagem e um farmacêutico, com tempo de formação profissional, em média, de 14 anos. Todos os participantes possuíam Curso de Pós-Graduação, sendo dez profissionais na área de Segurança do Paciente.

A análise dos dados identificou que, no que se refere à experiência de Identificação Correta do Paciente com transtorno mental agudo, a maioria dos participantes (82,3\%) não possuía em suas instituições um protocolo próprio voltado ao paciente.

As estratégias para a identificação relatadas pelos participantes originaram duas categorias: Adequação do Protocolo de Identificação proposto pelo MS; e Propostas a partir do Protocolo do MS. A primeira categoria foi construída por meio dos relatos dos participantes que orientam a identificação, de forma individualizada, dos portadores de transtornos mentais na fase aguda (42\%).

Quanto à segunda categoria, as estratégias citadas relacionam-se ao Protocolo do MS, sem a especificação das estratégias e protocolos voltados à Identificação Correta do Paciente com transtorno mental agudo (58\%), são estratégias generalistas, as quais não consideram atitudes voltadas ao contexto do paciente, podendo apresentar ações desorganizadas, devido à alteração do pensamento e da percepção ${ }^{8}$, sendo intrínseca a impossibilidade da auto identificação.

Destaca-se que, a análise dos dados também evidenciou as possíveis barreiras identificadas pelos participantes da pesquisa voltadas à Identificação Correta do Paciente com transtorno mental na fase aguda, através da construção de duas categorias: Fatores inerentes ao Paciente e Fatores inerentes ao Profissional.

A primeira categoria obteve o maior quantitativo de fatores $(62,2 \%)$, o que evidencia a especificidade destes pacientes como um desafio para a sua assistência segura e de qualidade, sendo composta pelos seguintes fatores: nível de consciência/orientação deste paciente; ausência de acompanhante; necessidade constante de vigilância; e retirada de pulseira de identificação pelo paciente. Tais elementos, também encontrados na literatura científica, atentam para a necessidade de maior vigilância do profissional de saúde diante da imprevisibilidade deste paciente 1 .

A individualidade deste paciente, distinta dos demais usuários, faz com que suas características sejam evidenciadas a fim de que as normas e diretrizes de Segurança do Paciente sejam adequadas a esta condição. O nível de consciência/orientação do paciente em apreço é a principal barreira potencializadora dos riscos aos quais estas pessoas estão expostas durante a assistência.

A ausência de acompanhante e a necessidade constante de vigilância foram citados pelos participantes como fatores que criam barreiras para a Identificação Correta do Paciente. Tais fatores aumentam a necessidade de observação deste paciente pela equipe, sendo necessária a vigilância intensiva, muitas vezes difícil de ser dispensada.

A segunda categoria, intitulada: "Fatores inerentes ao Profissional" referem-se às barreiras profissionais para a Identificação Correta do Paciente com transtorno mental agudo. Identificou-se que estas são voltadas para: Imposição da utilização da pulseira; Falta de adesão ao protocolo; Preconceito e falta de informação da equipe; e quantitativo reduzido de profissional de saúde mental. Os fatores profissionais são considerados fundamentais para uma prática segura e de qualidade, sendo importante sua identificação para construir estratégias de superação destas e potencializar a qualidade da assistência à saúde aos portadores de transtorno mental agudo.

A análise dos dados revelou que o processo de identificação ocorre da mesma forma dos outros pacientes, sem considerar as suas especificidades. Porém, é importante enfatizar que estabelecimento de barreiras frente ao referido processo, a necessidade de adequação na identificação correta a este paciente, por meio de protocolos, assim como, a qualificação profissional e reorganização dos serviços de saúde mental, de forma a minimizar os riscos envolvidos, contribuem para a segurança no cenário do cuidado.

Na terceira fase da pesquisa, participaram: 4 enfermeiros com especialização na área de Saúde Mental, sendo um com doutorado. Três participantes são do sexo feminino e 1 do masculino. A faixa etária variou de 40 a 76 anos. 0 tempo de formação profissional foi de 16 a 55 anos, e o período de experiência na área da saúde mental variou de 4 a 55 anos. 
Esta fase enfatiza a categoria "Identificação do Paciente no cotidiano de cuidados nos serviços de psiquiatria" construída, a partir da análise das falas dos participantes e abarcam: Falhas na Identificação Correta do Paciente com transtorno mental na fase aguda; Formas de identificação deste paciente; Especificidades do paciente com transtorno mental na fase aguda; Padronização dos cuidados na saúde mental; e Equipe interdisciplinar.

Quanto às falhas na identificação do paciente, foi unânime que, este sistema é falho dentro do cenário da psiquiatria, considerando a temática da Segurança do Paciente. Os participantes relataram que não existe a utilização da pulseira de identificação, preconizada pelo MS. Porém, entendem a necessidade de um sistema de identificação previamente definido para minimizar a ocorrência incidentes durante a assistência, principalmente na fase aguda, pois identificaram a especificidade deste paciente no cenário citado. Conforme observado na fala a seguir:

\section{[...] porque ele (o paciente) pode, de fato, estar desorientado. (Enfermeiro A)}

A falta de identificação do paciente em unidades de internação psiquiátrica também foi apontada em estudo desenvolvido com enfermeiros, com referência que, em unidades de internação psiquiátrica, não existem instrumentos para a identificação do paciente ${ }^{18}$.

No presente estudo, os participantes, especialistas na área de Saúde Mental, valorizaram as formas de identificação do portador de transtorno mental na fase aguda e suas especificidades como importantes fatores norteadores para a produção de práticas seguras. Na fala, a seguir, destaca-se a necessidade de avançar na identificação:

[...] A gente precisa de uma padronização. Eu acho que é necessário avançar em relação à identificação do paciente. (Enfermeiro B)

Quando questionados sobre como seria a melhor maneira de identificação do paciente com os sintomas agudos de doença mental, os participantes aventaram que, estando este sinalizado, identificado ou marcado, poderia ser gerada uma condição negativa no seu comportamento. Porém, a pulseira de identificação foi recomendada, pois esta não seria exclusividade deste paciente.

Outro aspecto importante, observado na análise deste estudo, foi a constatação dos participantes quanto à falta de um protocolo próprio para verificar a identidade do paciente, o que gera impacto na segurança do mesmo. Indivíduos na fase aguda, quando admitidos na emergência apresentam um comportamento imprevisível e nada cooperativo. Tais comportamentos foram descritos em estudos anteriores, quanto a demonstração de angústia e sofrimento deste paciente, por meio de agressividade e alteração do seu nível de consciência ${ }^{2,5}$.

Outra temática advinda da análise dos dados foi a necessidade da discussão da Identificação Correta do Paciente no contexto da atuação interdisciplinar no campo da Saúde Mental. Conforme os participantes do grupo focal, o processo de Identificação Correta do Paciente deve ser refletido com a equipe de profissionais que cuida do paciente com transtorno mental. Este processo de trabalho, para ser definido e colocado em prática, deverá contar com a aceitação e suporte de toda esta equipe, de modo que sejam definidos critérios e sugestões de acordo com a experiência de cada profissional envolvido.

A análise desenvolvida nesta investigação, ao evidenciar as contradições presentes entre as recomendações do MS quanto ao Processo de Identificação Correta do Paciente e o cotidiano de práticas nas unidades de psiquiatria, as barreiras encontradas e a importância de refletir sobre a temática em apreço, possibilitou elaborar uma ferramenta inovadora no protocolo de identificação do paciente com transtorno mental agudo. A partir das contradições encontradas, as quais identificam a importância da construção de ferramentas inovadoras para a identificação do paciente no quadro agudo; e as convergências presentes nas três fases do presente estudo são elencadas para a construção do protocolo em apreço. Assim, apontam-se, os pontos mais relevantes comuns às três fases do estudo.

A falha na comunicação do portador de transtorno mental é evidente nos estudos ${ }^{2,5}$. Esta condição está relacionada com a alteração do nível de consciência ou comprometimento cognitivo, peculiar em condições agudas ${ }^{1-4}$. Corroborado pelos profissionais da segurança onde $52 \%$ das respostas apontam para este problema. A literatura enfatiza a potencialização dos riscos destes pacientes ${ }^{3,5,17}$, sendo que todas as fases citaram como essencial a elaboração de um protocolo próprio.

Outra situação apontada pela revisão foi o estigma que acompanha o paciente com transtorno mental ${ }^{5,16}$. Os profissionais especialistas em Segurança do Paciente, participantes da primeira fase da pesquisa, concordam com esta condição. Todavia, sugeriram a utilização de uma foto na pulseira para facilitar a identificação deste paciente, devidos às suas características. Quanto aos profissionais da área da Saúde Mental, estes sugeriram a utilização da pulseira de identificação com o acréscimo do nome fictício caso o paciente assim se auto identifique.

Portanto, o protocolo elaborado inova ao propor uma pulseira de identificação diferenciada do padrão existente. Esta pulseira apresenta informações imagéticas e escritas, as quais tendem ultrapassar obstáculos encontrados pelo paciente e sua equipe assistencial, quando o usuário não consegue informar, com certeza, a sua própria identidade. 
Desta forma, aponta-se a articulação entre as três fases do estudo, com destaque às convergências advindas da análise dos dados. Assim, a Figura 2 apresenta os dados de maior relevância que foram elencados, conforme a análise dos dados das três fases do estudo.

\begin{tabular}{|lll|}
\hline \multicolumn{1}{|c}{ FASE 1 } & \multicolumn{1}{c|}{ FASE 2 } & \multicolumn{1}{c|}{ FASE 3} \\
Revisão Integrativa & $\begin{array}{l}\text { Abordagem a Profissionais de } \\
\text { Saúde em Segurança do Paciente }\end{array}$ & $\begin{array}{l}\text { Grupo Focal com Profissionais } \\
\text { de Saúde em Psiquiatria }\end{array}$ \\
\hline $\begin{array}{l}\text { Alteração do Nível de Consci- } \\
\text { ência/Falha na Comunicação. }\end{array}$ & $\begin{array}{l}52,6 \% \text { dos participantes apontou } \\
\text { como barreira a alteração do nível } \\
\text { de consciência deste paciente. }\end{array}$ & $\begin{array}{l}\text { Ausência de orientação deste } \\
\text { paciente. }\end{array}$ \\
Pacientes estigmatizados. & Pacientes estigmatizados. & $\begin{array}{l}\text { O estigma não é um problema } \\
\text { em potencial. }\end{array}$ \\
$\begin{array}{l}\text { Necessidade de um proto- } \\
\text { colo próprio. }\end{array}$ & $\begin{array}{l}\text { Necessidade de um protocolo pró- } \\
\text { prio, inserindo uma foto na pulsei- } \\
\text { ra de identificação. }\end{array}$ & $\begin{array}{l}\text { Necessidade de um protocolo } \\
\text { próprio. }\end{array}$ \\
\hline
\end{tabular}

FIGURA 2: Principais dados elencados, conforme cada análise da fase do estudo. Rio de Janeiro, RJ, Brasil, 2019.

\section{O Protocolo}

Trata-se de um protocolo próprio para a correta identificação do portador de transtorno mental agudo. Teve sua elaboração alicerçada no Protocolo de Identificação do MS, conduzida, portanto, por achados na literatura específica e informações de especialistas em segurança do paciente e saúde mental, onde as especificidades destes pacientes foram consideradas e adequadas conforme a preconização das políticas de segurança.

A pulseira elaborada é o grande diferencial do protocolo, a qual é considerada uma inovação de produto, por incorporar mudança em tecnologia já existente ${ }^{14}$. Possui além dos itens de identificação comuns, o código de barras e o QR-code, a ser utilizados em locais onde esta tecnologia já é utilizada. Como a proposta está baseada no Protocolo do MS, salienta-se que a serão aqui demonstrados somente os itens que sofreram adaptações.

A Figura 3 demonstra os principais itens do Protocolo de Identificação do paciente com transtorno mental na fase aguda.

\begin{tabular}{|c|c|c|}
\hline $\begin{array}{l}\text { Itens do } \\
\text { Protcolo }\end{array}$ & $\begin{array}{l}\text { Protocolo de Identificação } \\
\text { do Paciente, MS / } 2013\end{array}$ & $\begin{array}{l}\text { Protocolo de Identificação do Portador de } \\
\text { Transtorno Mental Agudo / } 2019\end{array}$ \\
\hline Finalidade & $\begin{array}{l}\text { Garantir a identificação de } \\
\text { qualquer paciente. }\end{array}$ & $\begin{array}{l}\text { Garantir a identificação do paciente com transtorno mental } \\
\text { agudo. }\end{array}$ \\
\hline Justificativa & $\begin{array}{l}\text { Custos advindos das falhas } \\
\text { da identificação. }\end{array}$ & $\begin{array}{l}\text { Custos advindos das falhas de identificação; fator } \\
\text { epidemiológico dos transtornos mentais. }\end{array}$ \\
\hline Abrangência & Serviços de saúde nacionais. & $\begin{array}{l}\text { Serviços de saúde que possuam de internação de forma } \\
\text { geral e emergência. }\end{array}$ \\
\hline \multirow[t]{2}{*}{ Intervenção } & $\begin{array}{l}\text { Dois identificadores (nome } \\
\text { completo e data de } \\
\text { nascimento). }\end{array}$ & $\begin{array}{l}\text { Acréscimo da foto e nome fictício na pulseira, além dos } \\
\text { identificadores já preconizados. A foto também deverá ser } \\
\text { inserida no prontuário e prescrição. }\end{array}$ \\
\hline & & 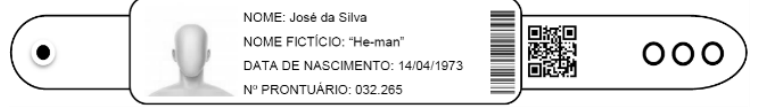 \\
\hline
\end{tabular}

Figura 3: Itens principais do Protocolo de Identificação do Paciente com transtorno mental na fase aguda comparados às recomendações do MS. Rio de Janeiro, RJ, Brasil, 2019.

Nota: Imagem produzida pela pesquisadora. 
Acréscimo de um centímetro na largura para comportar a foto, a qual terá o tamanho dois centímetros $(2 \mathrm{~cm})$ de altura por dois centímetros $(2 \mathrm{~cm})$ de largura, além de ter uma boa resolução e ser preferencialmente colorida. A outra alteração é a inclusão do campo "nome fictício". Além disso, destaca-se:

- Conteúdo: pulseira de cor branca, com bordas macias, lisas e arredondadas para a manutenção do conforto do paciente. Deverá possuir um fixador na extremidade para fechamento, e este não deve pressionar a pele.

- Dimensões:

- Comprimento total: 25 centímetros;

- Comprimento da área identificatória: 12 centímetros;

- Comprimento das alças de fechamento: 5 centímetros a menor e 8 centímetros a maior;

- Largura doa área identificatória: 3 centímetros;

- Largura das alças: 2 centímetros.

- Material: plástico impermeável, flexível, lavável e não alergênico.

\section{CONCLUSÃO}

A descrição da elaboração de protocolo para a Identificação Correta do Paciente com transtorno mental agudo identificou a importância da temática da Segurança do Paciente para o cuidado de qualidade em saúde neste grupo da população, e atenta para as práticas seguras junto à pacientes com transtorno mental agudo. Percebe-se a lacuna do conhecimento quanto às práticas desenvolvidas e à ausência de propostas de inovação quanto às metas relacionadas à segurança do paciente e as práticas em saúde e às particularidades relacionadas ao paciente com transtorno mental agudo.

Este estudo reafirma a importância da adequação das políticas de Segurança do Paciente em contextos individualizados. A implementação de medidas para a correta identificação desta clientela em qualquer cenário dos serviços de saúde, de acordo com as diretrizes de Segurança do Paciente, torna-se essencial. Todavia, tal prática, ainda é um desafio nos serviços de saúde. Assim, sugere-se, a necessidade de novos estudos que concretizem uma postura crítica frente às recomendações do MS no que se refere à Identificação Correta do Paciente voltado à identificação de formas individualizadas à cada grupo da população.

As limitações do estudo referem-se à discussão da Identificação Correta do Paciente com transtorno mental grave apenas com profissionais da área de Saúde Mental inseridos em unidades de saúde psiquiátricas, sendo necessário o diálogo com as equipes de saúde em unidades não psiquiátricas, por constituirem-se de cenários que tenham a possibilidade de recebê-los.

\section{REFERÊNCIAS}

1. Prado MF, Sa MC, Miranda L. The patient with severe mental disorders in general hospital: a literature review. Saúde Debate [Internet]. 2015 [cited 2018 Nov 07]; 39(spe):320-37. DOI: https://doi.org/10.5935/0103-1104.2015S005419

2. Daumit GL, McGinty EE, Pronovost P, Dixon LB, Guallar E, Ford DE et al. Patient safety events and harms during medical and surgical hospitalizations for persons with serious mental illness. Psychiatr. Serv. [Internet]. 2016 [cited 2018 Nov 11]; 67(10):1068-75. DOI: https://doi.org/10.1176/appi.ps.201500415

3. Higgins A, Doyle L, Downes C, Morrissey J, Costello P, Brennan M et al. There is more to risk and safety planning than dramatic risks: mental health nurses' riskassessment and safety-management practice. Int. J. Ment. Health Nurs. [Internet]. 2016 [cited 2018 Nov 10]; 25(2):159-70. DOI: https://doi.org/10.1111/inm.12180

4. Souza ACS, Bezerra ALQ, Pinho ES, Nunes FC, Caixeta CC. Patient safety in community mental health services: bibliometric study. Rev. enferm. UFPE on line. 2017 [cited 2018 Nov 10]; 11(Supl. 11): 4671-7. Available from: https://periodicos.ufpe.br/revistas/revistaenfermagem/article/viewFile/231208/25213

5. Kanerva A, Lammintakanen J, Kivinen, T. Nursing staff's perceptions of patient safety in psychiatric inpatient care. Perspect. Psychiatr. Care [Internet]. 2016 [cited 2018 Nov 10]; 52(1):25-31. DOI: https://doi.org/10.1111/ppc.12098

6. Capucho HC, Cassiani SHB. The need to establish a national patient safety program in Brazil. Rev. Saúde Pública [Internet]. 2013 [cited 2018 Feb 28]; 47(4):791-8. DOI: https://doi.org/10.1590/S0034-8910.2013047004402

7. Ministério da Saúde (Br). Portaria no 2.095/2013. Aprova os Protocolos Básicos de Segurança do Paciente. Diário Oficial da União. 2013 Sep 25[cited 2018 Feb 28]; (seção 1):113. Available from:

https://bvsms.saude.gov.br/bvs/saudelegis/gm/2013/prt2095_24_09_2013.html

8. Oliveira RM, Facina PCBR, Siqueira-Júnior Júnior AC. The reality of living with schizophrenia. Rev. bras. enferm. [Internet]. 2012 [cited 2018 Sep 11]; 65(2):309-16. DOI: http://dx.doi.org/10.1590/S0034-71672012000200017

9. Ministério da Saúde (Br). Documento de referência para o Programa Nacional de Segurança do Paciente. Brasília (DF): Ministério da Saúde. 2014 [cited 2018 Sep 11]; Available from: https://bvsms.saude.gov.br/bvs/publicacoes/documento_referencia_programa_nacional_seguranca.pdf

10. Krauzer IM, Dall'Agnoll CM, Gelbcke FL, Lorenzini E, Ferraz L. The construction of assistance protocols in nursing work. Rev. Min. Enferm. [Internet]. 2018 [cited 2020 Jul 9]; 22:e-1087. DOI: https://doi.org/10.5935/1415-2762.20180017 
11. Sales CB, Bernardes A, Gabriel CS, Brito MFP, Moura AA, Zanetti ACB. Standard operational protocols in professional nursing practice: use, weaknesses and potentialities. Rev. Bras. Enferm. [Internet]. 2018; [cited 2018 May 22]; 71(1):126-34. DOI: https://doi.org/10.1590/0034-7167-2016-0621

12. Lemos, CS, Poveda VB, Peniche ACG. Construction and validation of a nursing care protocol in anesthesia. Rev. Latino-Am. Enfermagem [Internet]. 2017 [cited 2019 Feb 25]; 25:e2952. DOI: https://doi.org/10.1590/1518-8345.2143.2952

13. Nicolini AB, Corrêa ACP, Medeiros RMK, Fraga JCAXO, Silva LA, Alvares AS. Development process of a protocol for nursing humanized care to the usual-riskchildbirth. Cienc. Cuid. Saude [Internet]. 2017 [cited 2018 Feb 12]; 16(4):1-7. DOI: https://doi.org/10.4025/cienccuidsaude.v16i4.36841

14. Organization for Economic Co-operation and Development. Oslo Manual 2018: guidelines for collecting, reporting and using data on innovation, 4th edition. Paris: OECD [Internet]. 2019 [cited 2019 Oct 15]; Available from: https://www.oecd.org/science/oslo-manual-2018-9789264304604-en.htm

15. Vantil FCS, Lima EFA, Figueiredo KC, Portugal FB, Sousa AI, Primo CC. Patient safety with mental disorder: developing management technologies for risk management. Esc. Anna Nery [Internet]. 2018 [cited 2019 Mar 15]; 22(4):e20170307. DOI: http://dx.doi.org/10.1590/2177-9465-ean-2017-0307

16. McGinty EE, Thompson D A, Pronovost PJ, Dixon LB, Guallar E, Ford DE et al. Patient, provider, and system factors contributing to patient safety events during medical and surgical hospitalizations for persons with serious mental illness. J. Ment. Nerv. Dis. [Internet]. 2017 [cited 2018 Nov 10]; 205(6):495-501. DOI: https://doi.org/10.1097/NMD.0000000000000675

17. Rise MB, Westerlund H, Bjørgen D, Steinsbekk A. Safely cared for or empowered in mental health care?: Yes, please. Int. J. Soc. Psychiatry [Internet]. 2014 [cited 2018 Nov 10]; 60(2):134-8. DOI: https://doi.org/10.1177/0020764012471278

18. Kelly T, Roper C, Elsom SE, Gaskin CJ. Identifying the 'right patient': nurse and consumer perspectives on verifying patient identity during medication administration. Int. J. Ment. Health Nurs. [Internet]. 2011 [cited 2018 Nov 03]; 20(5):371-9. DOI: https://doi.org/10.1111/j.1447-0349.2010.00739.x 